\title{
A Hybrid Approach for Robust and Precise Mobile Robot Navigation with Compact Environment Modeling
}

\author{
Nicola Tomatis ${ }^{\dagger}$, Illah Nourbakhsh ${ }^{\dagger}$, Kai Arras ${ }^{\dagger}$, Roland Siegwart ${ }^{\dagger}$ \\ ${ }^{\dagger}$ Autonomous Systems Lab \\ Swiss Federal Institute of Technology Lausanne (EPFL) \\ CH-1015 Lausanne \\ nicola.tomatis@epfl.ch \\ † The Robotics Institute \\ Carnige Mellon University \\ 5000 Forbes Avenue, Pittsburgh, PA 1513 \\ illah@ri.cmu.edu
}

\begin{abstract}
In this paper a new localization approach combining the metric and topological paradigm is presented. The main idea is to connect local metric maps by means of a global topological map. This allows a compact environment model which does not require global metric consistency and permits both precision and robustness. The method uses a 360 degree laser scanner in order to extract lines for the metric localization and doors, discontinuities and hallways for the topological approach. The approach has been widely tested in a $50 \times 25 \mathrm{~m}$ portion of the institute building with the new fully autonomous robot Donald Duck. 25 randomly generated test missions have been performed with a success ratio of $96 \%$ and a mean error at the goal point of $9 \mathrm{~mm}$ for an overall trajectory length of $1.15 \mathrm{~km}$. Future work will focus on a similar hybrid approach for simultaneous localization and automatic mapping.
\end{abstract}

\section{Introduction}

Perceiving the environment remains a fundamental task for autonomous mobile systems. More precisely, localization and mapping in an unmodified environment belongs to the basic skills for mobile robot applications. In many potential service tasks, the vehicle is operating in a structured or semi structured surrounding. This property can be exploited by using the structures as frequently and reliably recognizable landmarks for navigation. Topological, metric or hybrid navigation schemes can make use of different types of environment features on various levels of perceptual abstraction leading to different environment models.

Metric approaches (i.e. robot pose represented by $(x, y, \theta))$ based on Kalman filtering [7], [13] permit high accuracy and low complexity when using line based features from ultrasonic sensors [7], [13], CCD camera [5] or both laser scanner and CCD camera [15], [2]. Furthermore maps for this type of approach are very compact and directly extensible with feature information from different sensors. However the Kalman estimator is unimodal, therefore it has no mean to detect and recover from a lost situation (i.e. a situation where the robot cannot match features correctly anymore because the difference between the estimate and the real position is too high). Other approaches which do not suffer from this limitation are grid based and/or topological oriented. The success of Dervish at the 'AAAI robot contest' in 1994 [14], followed by formalization of the 'Partial Observable Markov Decision Process' (POMDP) [11] represented the start of this type of approach. In this case, the robot position is a grid cell, a topological position, or both, causing a non neglectable bound in the precision when comparing with Kalman based approaches. However this approach has lead to a metric, grid based method. The 'Markov localization' [10] permits both precision and multimodality by using small sized grid cells, but requires significant processing power and memory in order to update all the cells in the environment. More recently the 'Monte Carlo localization' [8] has proposed a more efficient alternative by using a sampling-based method that can represent arbitrary distributions.

In contrast to the above mentioned approaches, this paper proposes an integration of both the metric and topological paradigms, to gain the best characteristics of both universes. The system presented here embodies both a metric and a topological representation. The metric model consists of infinite lines that belong to the same place. These places are related to each other by means of a topological map that is composed of nodes and edges between nodes. Connections between a node and a place are a special case: Traveling along these edges causes a switch from topological to metric localization. Metric localization is done with a widely tested implementation of the Extended Kalman filter [2]. This approach guarantees high precision with low complexity and memory requirements. Topological navigation uses a POMDP based [3] state estimator. This permits efficient planning in the large, has an advantageous symbolic representation for man-machine interaction and is robust against lost situations thanks to its multimodality.

\section{Environment Modeling}

The model of the environment is characterized by two different levels of abstraction (see figure 1):

- Places are defined as a local metric map which allows navigation within the neighbourhood.

- To move from one place to another, the system switches from metric to topological when leaving the place, navigates by means of a topological state estimator within the global topological map and switches back to metric when reaching the goal place. 


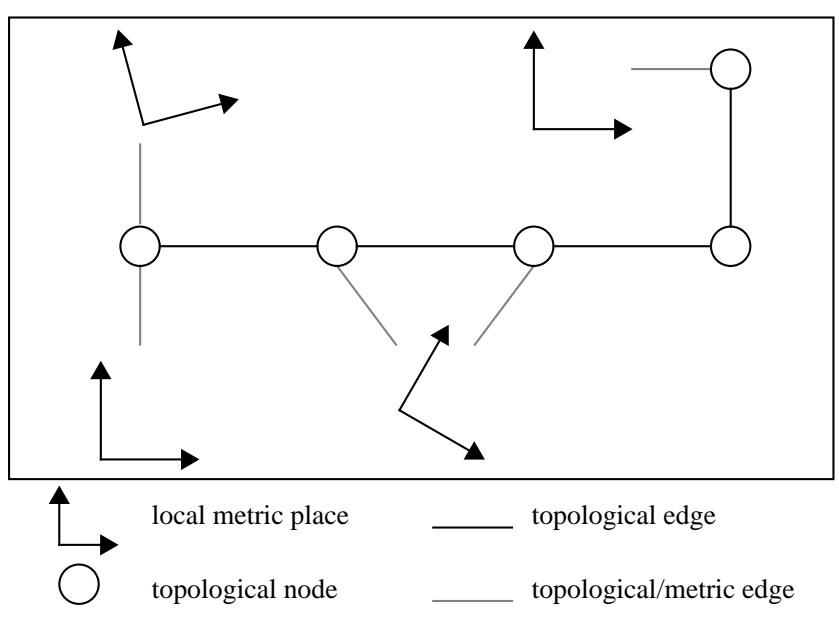

Figure 1: The environment is represented by places given by their metric maps and nodes representing topological states. When travelling from a node to a place, the system switches from topological to metric and vice-versa.

The only requirement for changing navigation model is to have a detectable metric feature when travelling from a topological node to a metric place. This permits the system to determine the transition point where the change from topological to metric has to be done and allows the initialization of the metric localization (i.e. relocation).

Although this is a very general and flexible approach, it is preferable to make some environment dependent assumptions/choices when implementing it on a real robot. In this case the experimental test bed is a part of the institute building. This environment is mainly composed of offices, meeting rooms and hallways. It seems an acceptable assumption to expect that the robot will have to be very precise in the rooms where most of its tasks have to be executed. While navigating in the large (i.e. hallways), precision with respect to the features is less important, but robustness and global consistency take an important role. Therefore the current implementation uses local metric maps for offices and rooms in general and a global topological map that connects them together. Because perception is not a goal of this paper, the features employed are as simple as possible and might be suboptimal, especially for landmark detection. Nevertheless they permit extensive testing of the proposed hybrid (metric/ topological) approach by means of a single sensor.

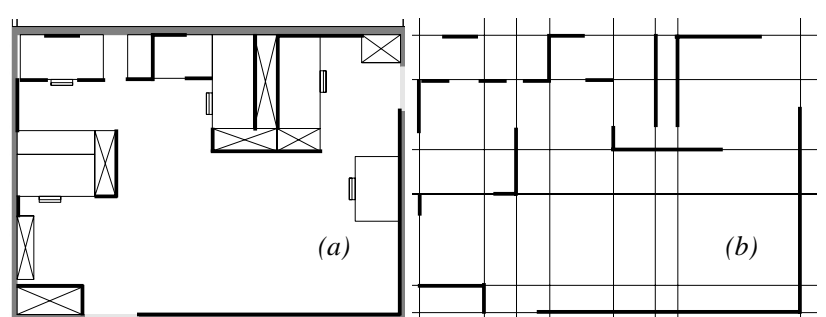

Figure 2: An office of the institute building (a) and the lines representing it in the local metric map (b). The black segments permit to see the correspondence between the two figures. This environment model is extremely compact with a memory requirement of about 10 bytes per $m^{2}$.

\subsection{Local Metric Maps}

The features used for metric environmental representation are infinite lines. They are less informative than line segments, but permit a very compact representation of structured geometric environments requiring only about 10 bytes per $m^{2}$. In figure 2 a typical office is shown with the lines used for its local metric map.

\subsection{Global Topological Map}

The topological map can be viewed as a graph. In each node the information about the visible features and the way to reach the connected nodes/places is stored. Only four directions of travel are employed: N, E, S, W. This implies the assumption that the environment is orthogonal, which is the case for most office buildings including the institute building where the robot operates. The above mentioned limitation is not an inherent loss of generality because it is only a simplification for the current implementation and not a general requirement of the algorithm.

The features for the topological representation are typical for hallways in office environments:

- Discontinuities perpendicular to the direction of travel in the hallway. They are characterized by the form ( $\mathrm{S}$ and Z) and the size of the step.

- Doors are used for state estimation and for model transition. Therefore they are fundamental for this approach.

- Hallways perpendicular to the direction of travel creating an intersection.

In figure 3 the graph representing the topological model is viewed for a portion of a hallway. Features are linked to the node from which they are best visible.

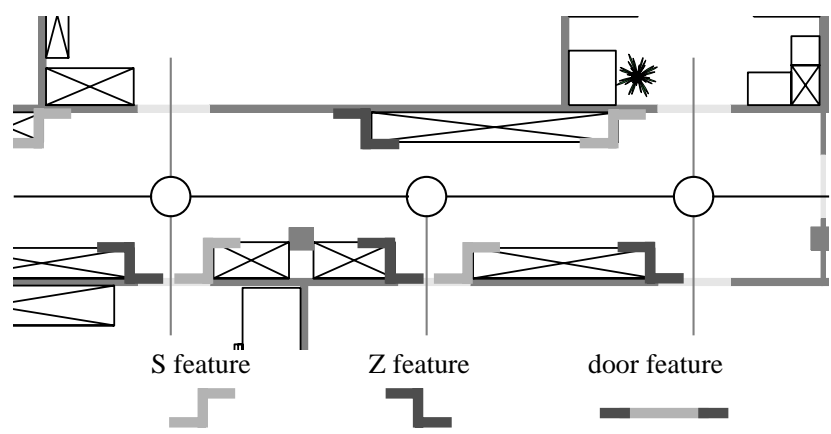

Figure 3: The topological map is represented by a graph. Each node contains the information about the visible features and the way to reach the adjacent nodes.

\section{Navigation}

Both environment models require a different navigation method with different characteristics. The metric approach permits a very precise positioning at the goal point whereas the topological method guarantees robustness against getting lost thanks to its multimodality. The estimation framework used for metric localization is the Kalman filter, while for topological navigation a partially observable Markov decision process (POMDP) model is used. 


\subsection{Metric Navigation}

For the Kalman filter, only a brief summary of a localization cycle is presented. Please refer to [13] for more details about its use in the context of mobile robot navigation. Furthermore some informations about uncertainty models and feature extraction are presented.

Odometry: Non-systematic odometry errors occur in two spaces: the joint space and the Cartesian space. With differential drive kinematics the joint space is two-dimensional (left and right wheel). Effects of wheel slippage, uneven ground and limited encoder resolution appear in this space. In [6] a physically well-grounded model for this kind of errors is presented starting from the uncertain input $u(k+1)=\left[\Delta d_{L}, \Delta d_{R}\right]^{T}$ where $\Delta d_{L}, \Delta d_{R}$ are the distances travelled by each wheel, and the diagonal input covariance matrix

$$
U(k+1)=\left[\begin{array}{cc}
k_{L}\left|\Delta d_{L}\right| & 0 \\
0 & k_{R}\left|\Delta d_{R}\right|
\end{array}\right]
$$

which relies on the assumption of proportionally growing variance per $\Delta d_{L}, \Delta d_{R}$ travelled. The odometry model for the first and second moment of the state vector $x$ $=(x, y, \theta)^{T}$ is then

$$
\begin{gathered}
x(k+1 \mid k)=f(x(k \mid k), u(k+1)) \\
P(k+1 \mid k)=\nabla f_{x} P(k \mid k) \nabla f_{x}^{T}+\nabla f_{u} U(k+1) \nabla f_{u}^{T}
\end{gathered}
$$

where $f($.$) uses a piecewise linear approximation, P(k \mid k)$ is the state covariance matrix of the last step and $\nabla f_{x, u}$ is the Jacobian of $f($.$) with respect to the uncertain vectors$ $x(k \mid k)$ and $u(k+1) . k_{L}$ and $k_{R}$ are constants.

Effects of external forces (mainly collisions) occur in this Cartesian space. Because it is difficult to identify parameters for models taking these non-systematic errors into account, in this work only the above presented model is used.

Feature Extraction: The line model is

$$
\rho \cos (\varphi-\alpha)-r=0
$$

where $(\rho, \varphi)$ is the raw measurement and $(\alpha, r)$ the model parameters. $\alpha$ is the angle of the perpendicular to the line, $r$ its length. The used extraction algorithm has been described in [1]. It differs from the widely used recursive split-andmerge technique in the segmentation criterion: Instead of using a line specific decision on a single point, it decides on a model independent criterion on a group of points, so that multiple segments which lie on the same physical object are merged for particular precise re-estimates of the line position.

\section{Localization cycle:}

State Prediction. The state $\hat{x}(k+1 \mid k)$ and its associated covariance $P(k+1 \mid k)$ are determined from odometry based on the previous state moments $\hat{x}(k \mid k)$ and $P(k \mid k)$.

Observation. The extracted feature parameters $(\alpha, r)^{T}$ constitute the vector of observations $z(k+1)$ and its associ- ated observation covariance matrix $R(k+1)$. Since measurement errors features are independent, $R(k+1)$ is blockwise diagonal. That means that all subsequent equations operate with $2 \times 2$-matrices.

Measurement Prediction. The modeled features in the map, ${ }^{W} m$, get transformed into the frame of the observations. The first moments are computed by $\hat{z}(k+1)=$ $h\left(\hat{x}(k+1 \mid k),{ }^{W} m\right)$ where $h($.$) is the nonlinear measure-$ ment model (the global-to-local transform). Error propagation is done by a first-order approximation which requires the Jacobian $\nabla h$ with respect to the state prediction $\hat{x}(k+1 \mid k)$.

Matching. Because the observation covariance matrix $R(k+1)$ is blockwise diagonal, the matched pairings can be integrated in a manner which is advantageous for filter convergence: Each pairing is integrated according to its quality in an iterative procedure: $(i)$ matching of the current best pairing, (ii) estimation and (iii) re-prediction of features not associated so far. This procedure has also been used in [15]. The quality of a pairing of prediction $\hat{z}^{[j]}$ and observation $z^{[i]}$ is smallest observational uncertainty - not smallest Mahalanobis distance like in [15]. This renders the matching robust against small spurious and uncertain segments which have small Mahalanobis distances. The 'current best' pairing $\left(z^{[i]}, \hat{z}^{[j]}\right)$ is then the observation $z^{[i]}$ with $\operatorname{trace}\left(R^{[i]}\right)=\min _{i}$ which satisfies the validation test

$$
\left(z^{[i]}-\hat{z}^{[j]}\right) S_{i j}^{-1}\left(z^{[i]}-\hat{z}^{[j]}\right)^{T} \leq \chi_{\alpha, n}^{2}
$$

where $S_{i j}$ is the innovation covariance matrix of the pairing and $\chi_{\alpha, n}^{2}$ a number taken from a $\chi^{2}$ distribution with $n=2$ degrees of freedom. $\alpha$ is the level on which the hypothesis of pairing correctness is rejected.

Estimation. Successfully matched observation and predictions yield the innovations $v(k+1)=z(k+1)-\hat{z}(k+1)$ and their innovation covariance matrix $S(k+1)=$ $\nabla h P(k+1 \mid k) \nabla h^{T}+R(k+1)$. Finally, with the filter equations

$$
\begin{gathered}
W(k+1)=P(k+1 \mid k) \nabla h^{T} S^{-1}(k+1) \\
\hat{x}(k+1 \mid k+1)=\hat{x}(k+1 \mid k)+W(k+1) v(k+1) \\
P(k+1 \mid k+1) \\
=P(k+1 \mid k)-W(k+1) S(k+1) W^{T}(k+1)
\end{gathered}
$$

the posterior estimates of the robot pose and associated covariance are computed.

Local Navigation: Local navigation is done by a motion control algorithm. It plays the role of both position controller and obstacle avoidance: it reaches the given $(x, y, \theta)$ or $(x, y)$ goal by planning a collision free path (with respect to the current local data), and reacting to the dynamic environment either by merely replanning the path or by changing heading direction and replanning when an object appears in front of the robot. 


\subsection{Topological Navigation}

A detailed description of the POMDP approach is given in [3] and [11]. In this paper only the state estimator and the heading estimator are presented. Details about the control strategy and the local navigation are discussed below.

Feature Extraction: Features $S$ and $Z$ (figure 3) are line based and thus extracted by using the information from the line extractor presented in section 3.1. Doors are extracted by combining information from raw data (opening detection) and line extractor (open door). Hallways are modeled as openings and characterized by the distance between the node and the end of the hallway, which is detected by means of a perpendicular line.

Position Estimator: Given a finite set of environment states $S$, a finite set of actions $A$ and a state transition model $T$, the model can be defined by introducing partial observability. This includes a finite set $O$ of possible observations and an observation function $O S$, mapping $S$ into a discrete probability distribution over $O . T\left(s, a, s^{\prime}\right)$ represents the probability that the environment makes a transition from state $s$ to state $s^{\prime}$ when action $a$ is taken. $O S(o, s)$ is the probability of making an observation $o$ in state $s$. The probability of being in state $s^{\prime}$ (belief state of $s^{\prime}$ ) after having made observation $o$ while performing action $a$ is then given by the following equation:

$$
S E_{s^{\prime}}(k+1)=\frac{O S\left(o, s^{\prime}\right) \sum_{s \in S} T\left(s, a, s^{\prime}\right) S E_{s}(k)}{P(o \mid a, S E(k))}
$$

where $S E_{s}(k)$ is the belief state of $s$ at the last step, $S E(k)$ is the belief state vector of last step and $P(o \mid a, S E(k))$ is a normalizing factor.

Heading Estimator: The position estimator calculates the probability of being in a state, but does not take into account the heading of the robot. Nevertheless to work properly, the position estimator has to know the robot direction in order to distinguish between the four directions of travel allowed by the topological model (see section 2.2). This is done by a weighted mean of each observed line that is either horizontal or vertical with respect to the environment. The success of this method is due to the fact that in a rectilinear office building the vast majority of flat surfaces are aligned with the principal building directions. Lines are matched by means of the validation test $\left(z^{[i]}-\hat{z}^{[j]}\right) S_{i j}^{-1}\left(z^{[i]}-\hat{z}^{[j]}\right)^{T} \leq \chi_{\alpha, n}^{2}$, where prediction $\hat{z}^{[j]}$ is directly the odometry state vector variable $\theta$. In this case, $\chi_{\alpha, n}^{2}$ is a number taken from a $\chi^{2}$ distribution with $n=1$ degrees of freedom. This can be viewed as a Kalman filter for heading only.

Control Strategy: Since it is computationally intractable to compute the optimal POMDP control strategy for a large environment [3], simple suboptimal heuristics are introduced. For the system presented here the most likely state policy has been adopted: the world state with the highest probability is found and the action that would be optimal for that state is executed. For unconfident states (calculated by means of the confidence function $C(S E(k)))$ a further heuristic is introduced: if the optimal action is the same for all the states with high probability, the action will be executed directly, otherwise the system searches for the best acceptable action for all the high probability states that permits information gain for the state estimator (ex.: invert direction and follow mid line).

Local Navigation: In this environment, topological navigation takes place in long hallways. Therefore only a few actions are needed. Follow mid line, and wall following permit the robot to navigate in the global environment. Furthermore the door find and passing action is used for changing from topological to metric. All actions are implemented by using the motion control algorithm presented in section 3.1.

\subsection{Switching Model}

Because the topological navigation method is multimodal, the confidence before switching to the unimodal metric navigation is very critical. In contrast to pure topological navigation, a false state when entering a local metric place would cause the robot to search a goal position which is false and could even be inaccessible. Therefore a door find and passing action is executed only when the estimator is highly confident: $C(S E(k))>c$, where $c$ is a constant defined by experience. If it is not the case, the best action for gaining more information is taken (Control Strategy in section 3.2).

When switching from topological to metric another important problem has to be faced: the Kalman filter has to be initialized (i.e. $\hat{x}(k \mid k)$ and $P(k \mid k)$ are unknown). Such a task is often referred to as relocation problem. Although this is a complex problem, it can be simplified for this approach. As explained in chapter 2 , a detectable metric feature (doors in this case) between a node and a place permits knowing when to switch and gives an approximation of the robot position with respect to the local metric map. The first two moments of the measure are used to initialize the Kalman filter and permit a fast convergence of the filter.

Changing from metric to topological reduces to a metric navigation to the initialization position of the robot for the current local place. There the initialization of the states of the graph representing the global map takes place.

\section{Experimental Results}

For the experiments, a fully autonomous mobile vehicle has been used. Donald Duck (figure 4) is connected via radio ethernet only for data visualization via web and data logging for statistical purposes. The approach has been tested during the day, under normal conditions, in the portion of the institute building shown in figure 5. This environment is not only complex but also highly dynamic due to the presence of the coffee room and the secretariat, which raise the flow of humans in the hallways. 


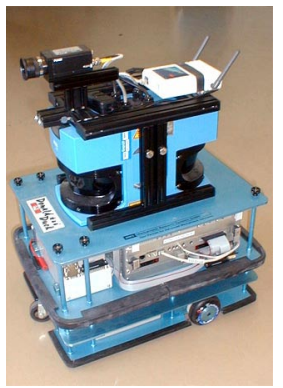

Figure 4: The autonomous robot Donald Duck. Its controller consists of a VME standard backplane with a Motorola PowerPC 604 microprocessor clocked at $300 \mathrm{Mhz}$. Among its peripheral devices, the most important are the wheel encoders, a $360^{\circ}$ laser range finder and a grey-level CCD camera (not used here).

\subsection{Experiments}

Test missions are generated randomly where the start and end point correspond to the crosses of figure 5. The robot is localized with respect to the local metric map at the start position. By leaving the room it switches to topological navigation. When it reaches the goal place, it initializes the Kalman filter and navigates metrically to the goal point. There the position error is measured.

\subsection{Results}

Donald performed 25 randomly generated mission and achieved an overall success rate of $96 \%$. A mission is classified as successful when the robot reaches the goal point and is localized with respect to the local metric map in the goal place. The overall distance travelled is $1.15 \mathrm{~km}$. Note that $0.95 \mathrm{~km}$ have been done in topological mode, which is multimodal and therefore robust against lost situations. The 'mean error at goal point' is the average of the differences between the robot estimate and its real position at goal point. It is only $9 \mathrm{~mm}$ demonstrating the accuracy of the Kalman approach. When the robot has to use the second heuristic for the control strategy (see section 3.2), it encountered an 'unconfident state'. This appears in $23 \%$ of the estimates, which seems relative high, but the control strategy always solves them. Nevertheless that remains suboptimal because in four missions it causes robot navigation for information gaining only. In one experiment the robot entered the wrong local place because noise in its perception caused a confident false state where the model change had to take place.

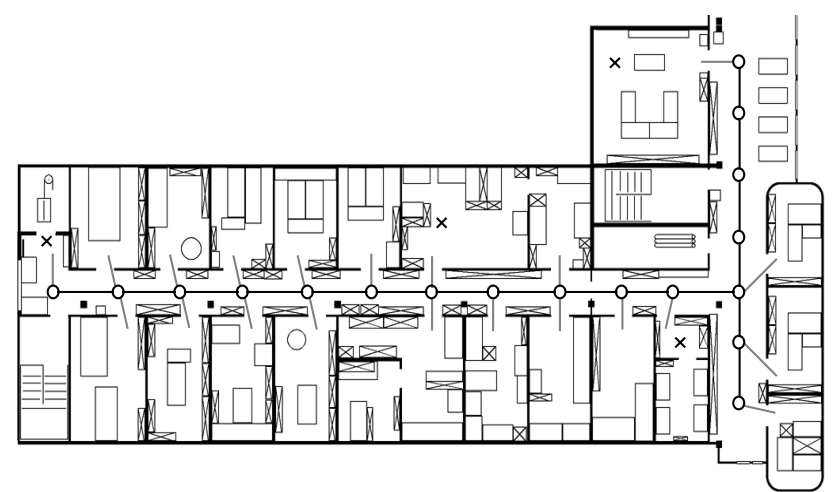

Figure 5: The map of the test environment with the graph representing the topological map. The crosses represent the start or end of the randomly generated test missions.

\begin{tabular}{c|c}
\hline number of missions & 25 \\
\hline success rate & $96 \%$ \\
\hline number of state estimates & 788 \\
\hline unconfident state rate & $23 \%$ \\
\hline total travel distance & $1.15 \mathrm{~km}$ \\
\hline topological travel distance & $0.95 \mathrm{~km}$ \\
\hline metric travel distance & $0.2 \mathrm{~km}$ \\
\hline mean error at goal point & $9 \mathrm{~mm}$ \\
\hline
\end{tabular}

Table 1: Summary of the experiments. The results demonstrate the feasibility of this hybrid approach for office environments.

\section{Related Work}

Successful navigation of embedded systems for real applications relies on the precision that the vehicle can achieve, the capacity of not getting lost and the practicability of their algorithms on the limited resources of the autonomous system. This problem has been faced with approaches that can be separated into two categories: metric (geometric or feature based) and topological.

Metric approaches using Kalman filtering can be very precise [15], [2], they allow compact environment modeling and therefore on-board calculation with today's processors power. They have also proven their robustness in large, application like experiments [2]. Nevertheless their solution remains unimodal. This means that an unmodeled event (i.e. collision) could cause a lost situation from which the system is unable to recover. Other metric approaches, like those based on Markov localization [10], are multimodal (i.e. robust against lost situations) and precise when small grids are used, but require off-board processing because of their computational complexity. Even if the 'Monte Carlo localization' [8] proposes a promising improvement for the efficiency, a trade-off between precision and real-time embedded computability remains to be found as soon as multimodality is introduced in metric approaches.

On the other hand, topologically based methods are very robust, but lack in precision. The main idea itself of only using the topology of an environment [12] causes a loss of the metric paradigm which is indispensable to guarantee precision. Nevertheless the robustness of these approaches has been often proven [14]. POMDP methods are used and improved for optimal and computational acceptable decision making [3]. By combining this approach with metric information, the lack in precision can be overcome.

Metric and topological approaches are converging to a hybrid solution combining the best characteristics of both worlds. In [4] a basically metric based approach with absolute localization is extended to include a localization relative to a local reference frame. This results in a two level abstraction which remains metric, but embodies a topology in the constellation of the local frames. In [16] the approach consists in extracting a topological map from a grid map with a 
Voronoi based method. In this case the topological navigation permits a gain in efficiency with respect to the metric grid based navigation. In [9] an approach for both global localization and position tracking using a topological map augmented with metric information is presented. The method is based on histogram matching of ultrasonic range data.

For this system a natural integration of the metric and topological paradigm is proposed. The approaches are completely separated into two levels of abstraction. Metric maps are used only locally for structures (rooms) that are naturally defined by the environment. There, a fully metric localization and navigation method is adopted. Topological navigation and localization are used to connect the local metric maps that can be far away from each other. This intuitive way to gain in robustness while maintaining precision at the goal/task point is similar to the behavior humans have: when going to a room where a task has to be done, the human localizes itself roughly with respect to doors, hallways, crosses and so on, but in the goal room he measures exactly his position with respect to the coffee machine where he has to put his cup. Even the heuristics presented in section 3.2 take inspiration from mankind: if the person searching for the coffee machine isn't sure about the door he has to enter, he will go back some meters or continue in the hallway to gain information in order to be sure not to enter in the office of the big boss which is the next one after the coffee room.

A further aspect of this approach is the emphasis in compactness of the environment representation. This permits to be very efficient when calculating the robot position. Figure 2 shows an office which is metrically modeled with only 14 lines, while the whole environment is represented topologically by less than 20 nodes (figure 5).

\section{Conclusion and Outlook}

This paper presents a new hybrid approach for localization. The metric and topological parts are completely separated into two levels of abstraction. Together they permit a very compact and computationally efficient representation of the environment for mobile robot navigation. Furthermore this combination permits both precision with the non-discrete metric estimator and robustness by means of the multimodal topological approach. The success rate over the 1.15 $\mathrm{km}$ of the 25 tests missions is $96 \%$, meaning that only one mission was not fulfilled. The mean error at the goal point is only $9 \mathrm{~mm}$. The $23 \%$ of unconfident states are uncritical in the experiments, nevertheless they cause a loss of time when travelling for gaining further information.

Future work will focus on the choice of a better suited perception for the topological part by evaluating other landmarks and adding the information from a CCD camera. This could allow a reduction of unconfident states. Furthermore success could be guaranteed for each mission by employing the topological approach in parallel to the metric estimator when navigating in metric places.

Moreover the main goal for the future is the implementa- tion of such a hybrid approach for simultaneous localization and map building.

\section{Acknowledgements}

Thanks to Daniel Huber, Dave Hershberger and Nicholas Roy at Robotics Institute, CMU, and Björn Jensen at ASL, EPFL, for their valuable feedback about this work.

\section{References}

[1] Arras, K.O. and R.Y. Siegwart (1997). Feature Extraction and Scene Interpretation for Map-Based Navigation and Map Building, Proceedings of SPIE, Mobile Robotics XII, Vol. 3210 .

[2] Arras, K. O., N. Tomatis, et al. (2000). "Multisensor On-theFly Localization: Precision and Reliability for Applications". To appear in Journal of Robotics and Autonomous Systems.

[3] Cassandra, A. R., L. P. Kaelbling, et al. (1996). Acting under Uncertainty: Discrete Bayesian Models for Mobile-Robot Navigation. IEEE International Conference on Robotics and Automation, Osaka, Japan.

[4] Castellanos, J. A., M. Devy, et al. (2000). Simultaneous Localization and Map Building for Mobile Robots: A Landmark-based Approach. IEEE International Conference on Robotics and Automation (ICRA), San Francisco.

[5] Chenavier, F. and J.L. Crowley (1992). Position Estimation for a Mobile Robot Using Vision and Odometry, IEEE InternationalConferenceonRoboticsandAutomation,Nice,France.

[6] Chong, K.S. and L. Kleeman (1997). Accurate Odometry and Error Modelling for a Mobile Robot, IEEE International Conference on Robotics and Automation, NM, USA.

[7] Crowley, J.L. (1989). World Modeling and Position Estimation for a Mobile Robot Using Ultrasonic Ranging, IEEE International Conference on Robotics and Automation, Scottsdale, AZ.

[8] Dellaert F., D. Fox et al. (1999). Monte Carlo Localization for Mobile Robots, IEEE International Conference on Robotics and Automation, Detroit Michigan.

[9] Duckett, T. and U. Nehmzow (1999). Knowing your place in real world environments. Third European Workshop on Advanced Mobile Robots (Eurobot 99), Zurich, Switzerland.

[10] Fox, D. (1998). Markov Localization: A Probabilistic Framework for Mobile Robot Localization and Navigation. Institute of Computer Science III. Bonn, Germany, University of Bonn.

[11] Kaelbling, L. P., M. L. Littman, et al. (1995). Partially Observable Markov Decision Processes for Artificial Intelligence. Int. Workshop, Reasoning with Uncertainty in Robotics, RUR '95, Amsterdam, Netherlands, Springer.

[12] Kuipers, B. J. and Y. T. Byun (1987). A qualitative approach to robot exploration and map-learning. Workshop on Spatial Reasoning and Multi-Sensor Fusion, Los Altos, CA, USA, Morgan Kaufmann.

[13] Leonard, J.J. and H.F. Durrant-Whyte (1992). Directed Sonar Sensing for Mobile Robot Navigation, Kluwer Academic Publishers.

[14] Nourbakhsh, I. (1998). Dervish: An Office-Navigating Robot. Artificial Intelligence and Mobile Robots. D. Kortenkamp, R. P. Bonasso and R. Murphy, The AAAI Press/ The MIT Press: 73-90

[15] Pérez, J.A., J. A. Castellanos et al. (1999). Continuous Mobile Robot Localization: Vision vs. Laser, IEEE International Conference on Robotics and Automation, Detroit.

[16] Thrun, S. and A. Bücken (1996). Integrating grid-based and topological maps for mobile robot navigation. National Conference on Artificial Intelligence, AAAI, Portland, OR, USA. 